\title{
Editorial
}

\section{Guidance on That Damned Elusive Orbitopathy of Graves' Disease}

\author{
Peter Laurberg \\ Department of Endocrinology, Aalborg University Hospital, and Department of Clinical Medicine, \\ Aalborg University, Aalborg, Denmark
}

Graves' disease with orbitopathy (GO) may severely impair life if it is not treated appropriately. In his article on 'bulging eyes', Basedow [1] described the 'unspeakable pain' and blindness secondary to keratitis in one of his GO patients. This was around 1840 when no therapy for the disease was available. Recently, another German study described some important consequences in modern society: the considerable short- and long-term work disability and public expenses caused by diplopia in patients with GO [2].

Thus, effective and safe therapy of GO is much needed. In the current issue of European Thyroid Journal, the EUGOGO group of collaborating experts in the field have updated their former consensus report [3] and developed it into a regular set of ETA guidelines [4]. The presentation is systematic and easily understandable, and it is a much welcomed contribution in this difficult field of clinical medicine.

Unfortunately, even with these guidelines in hand, response to therapy of moderate and severe GO is often less than satisfactory, and overall the disease can be a significant burden for the patient as well as for the health care providers involved.

A major problem in development of good therapies for GO is our incomplete understanding of the basic mecha- nisms of the disease as well as the cause of several of its clinical characteristics. For example, in my head, Graves' disease is a common autoimmune disease that may affect a number of organs and may have various manifestations in each organ. It thus follows that patients with GO suffer from Graves' disease whether they are hyper-, hypo- or euthyroid. However, many authors narrow Graves' disease down to include patients with hyperthyroidism only and state that GO (or 'thyroid-associated ophthalmopathy' or 'endocrine exophthalmus', or one of several other names) may also develop in other disorders besides Graves' disease. Maybe international consensus on the definition of Graves' disease would be of value.

Many ideas have been brought forward in attempts to understand GO, from thyroid-to-orbit transport of thyroglobulin via lymphatic connections as proposed by Kriss [5], to quite complicated models of TSH-receptor and IGF1-receptor interactions that involve fibrocytes from bone marrow and a series of immune system components [6]. At the recent ITC15 in Orlando, much research was focused on orbital fibroblasts and their production of glucosaminoglycans being the central culprit, but no pathogenic model has completely explained the clinical characteristics of the disorder.

\section{KARGER}

E-Mail karger@karger.com www.karger.com/etj
Peter Laurberg, MD

Department of Endocrinology and Medicine

Aalborg Hospital, Aalborg University

DK-9000 Aalborg (Denmark)

E-Mail peter.laurberg@ rn.dk 
At the time of diagnosis of Graves' hyperthyroidism, $20-30 \%$ of patients have GO that is mostly mild [7]. The age distribution of these hyperthyroid patients is the same irrespective of eye signs being present or not, and most often the eye signs disappear when the hyperthyroidism is treated [8]. A subset (around 5\% of all Graves' patients) develops a more severe GO, mostly after the debut of hyperthyroidism, but this rarely happens in young patients [9]. Immune responses change with aging in parallel with a fall in the activity of the adaptive and an activation of the innate immune system, but the exact mechanism behind the overrepresentation of middle aged and elderly patients among those who develop moderate and severe $\mathrm{GO}$ is unknown.

Another detail is the difference that may be present in the clinical appearance of patients. The more severe cases of GO may present with much inflammation and with a high clinical activity score [10], but some cases are different. Many patients with diplopia have more isolated effects on external eye muscles, and often signs of orbital inflammation are less severe [11]. We do not understand the mechanism behind this difference, and whether therapeutic approaches should be different. We also do not understand why only some patients experience an effect on their eyelid muscles, or why some patients have entirely unilateral disease. A common hypothesis is that mechanical factors are involved, and one study speculated that sleeping mostly on one side might increase disease activity on that side by leading to a higher orbital pressure. However, such a mechanical cause was not verified [12]. Similarly, we do not understand why the patterns of effects on muscles differ between patients. Even though the inferior and medial rectus muscles are the most commonly affected, patients may occasionally experience some isolated effects on other external eye muscles.

Considering the clinical treatment care of patients with GO, some expansion of guidance has been taking place from the previous consensus statement published in 2008 [3]. In particular, new controlled studies have detailed the practical use of intravenous steroids. Moreover, rituximab has been added to the list of possible therapies in cases that do not respond to other types of therapy, even though the evidence for such use is limited and somewhat controversial. A protective role of selenium supplements against progression of mild GO has been demonstrated in one controlled study.

This is a somewhat disappointing lack of development in medical therapy. Some other severe autoimmune diseases have benefitted considerably from new so-called 'biological therapies' which interfere with the immune system. Why has this not happened to the same degree in GO? A major reason is that moderate and severe GO is not a common disorder and that disease presentation is rather diverse between patients, making controlled studies more difficult to perform.

Unfortunately, knowledge on the positive effects of a certain drug from therapy of other autoimmune diseases does not guarantee an effect in GO. Autoimmune diseases are rather different in their responses to many factors. For example, Graves' disease and other autoimmune thyroid diseases predominately affect women. On the other hand, there is little sex difference in type 1 diabetes, and ankylosing spondylitis is mostly seen in men [13]. Similarly, pregnancy and the postpartum time are associated with much variation in incidence of Graves' disease, but they affect inflammatory bowel disease much less [14].

Accordingly, the way forward is to organize prospective controlled studies of new immune modulating drugs in GO, and not to forget attempts to prevent development of GO. Recently, a higher risk of developing GO after radioiodine therapy of hyperthyroidism was again observed [15]. Efforts to improve prevention and therapy of GO have been optimally executed in a collaboration with patients, which may lead to more focus on aspects such as psychological therapy to improve well-being than on new drugs to improve more hardcore functional end points [16].

Hopefully, the EUGOGO group and other investigators will manage to organize basic and clinical studies in a way that leads to new and better therapies of GO in the not too distant future.

\section{Disclosure Statement}

The author has nothing to disclose.
References
1 Basedow CA: Die Glotzaugen. Wochenschr Gesamte Heilk 1848;49:769-777.
2 Ponto KA, Merkesdal S, Hommel G, Pitz S, Pfeiffer N, Kahaly GJ: Public health relevance of Graves' orbitopathy. J Clin Endocrinol Metab 2013;98:145-152.
3 Bartalena L, Baldeschi L, Dickinson A, Eck- stein A, Kendall-Taylor P, Marcocci C, et al: Consensus statement of the European Group on Graves' orbitopathy (EUGOGO) on man- agement of GO. Eur J Endocrinol 2008;158: 273-285. 
4 Bartalena L, Baldeschi L, Boboridis K, Eckstein A, Kahaly GJ, Marcocci C, Perros P, Salvi $\mathrm{M}$, Wiersinga WM; on behalf of the European Group on Graves' Orbitopathy (EUGOGO): The 2016European Thyroid Association/ European Group on Graves' Orbitopathy Guidelines for the Management of Graves' Orbitopathy. Eur Thyroid J 2016;5:9-26

5 Kriss JP: Radioisotopic thyroidolymphography in patients with Graves' disease. J Clin Endocrinol Metab 1970;31:315-323.

6 Wang Y, Smith TJ: Current concepts in the molecular pathogenesis of thyroid-associated ophthalmopathy. Invest Ophthalmol Vis Sci 2014;55:1735-1748.

7 Laurberg P, Nygaard B, Andersen S, Carle A, Karmisholt J, Krejbjerg A, et al: Association between tsh-receptor autoimmunity, hyperthyroidism, goitre, and orbitopathy in $208 \mathrm{pa}-$ tients included in the Remission Induction and Sustenance in Graves' Disease Study. J Thyroid Res 2014;2014:165487.
-8 Tanda ML, Piantanida E, Liparulo L, Veronesi G, Lai A, Sassi L, et al: Prevalence and natural history of Graves' orbitopathy in a large series of patients with newly diagnosed Graves' hyperthyroidism seen at a single center. J Clin Endocrinol Metab 2013;98:14431449.

9 Laurberg P, Berman DC, Bulow Pedersen I, Andersen S, Carle A: Incidence and clinical presentation of moderate to severe Graves' orbitopathy in a Danish population before and after iodine fortification of salt. J Clin Endocrinol Metab 2012;97:2325-2332.

10 Mourits MP, Prummel MF, Wiersinga WM, Koornneef L: Clinical activity score as a guide in the management of patients with Graves' ophthalmopathy. Clin Endocrinol 1997;47: 9-14.

11 Laurberg P, Berman DC, Pedersen IB, Andersen S, Carle A: Double vision is a major manifestation in moderate to severe Graves' orbitopathy, but it correlates negatively with inflammatory signs and proptosis. J Clin Endocrinol Metab 2015;100:2098-2105.
12 Wiersinga WM, Bleumink M, Saeed P, Baldeschi L, Prummel MF: Is sleeping position related to asymmetry in bilateral Graves' ophthalmopathy? Thyroid 2008;18:541-544.

13 Ngo ST, Steyn FJ, McCombe PA: Gender differences in autoimmune disease. Front Neuroendocrinol 2014;35:347-369.

14 Andersen SL, Olsen J, Carle A, Laurberg P: Hyperthyroidism incidence fluctuates widely in and around pregnancy and is at variance with some other autoimmune diseases: a Danish population-based study. J Clin Endocrinol Metab 2015;100:1164-1171.

15 Stein JD, Childers D, Gupta S, Talwar N, Nan B, Lee BJ, et al: Risk factors for developing thyroid-associated ophthalmopathy among individuals with Graves' disease. JAMA Ophthalmol 2015;133:290-296.

16 Perros P, Dayan C, Dickinson J, Ezra DG, Hickey JL, Hintschisch C, et al: Future research in Graves' orbitopathy: from priority setting to trial design through patient and public involvement. Thyroid 2015;25:11811184. 